\title{
SIMULATION ET DISSIMULATION CHEZ L'HÉROÏNE-NARRATRICE DE LA VIE DE MARIANNE ${ }^{l}$ DE MARIVAUX
}

\author{
Jacques GUILHEMBET \\ Université Paris Sorbonne-Paris IV
}

\begin{abstract}
En): This paper analyzes the forms and effects of simulation and dissimulation in Marivaux's novel The Life of Marianne, or the Adventures of the Countess of *** (La Vie de Marianne, 1731-42). In the diegesis, young Marianne is a dissembler inclined to playacting on the world stage. She often acts coquettishly, easily manipulates some of her interlocutors, and gives in to romantic storytelling when fantasizing about her origins.

In keeping with such a trend, the autodiegetic narrator also resorts to dissimulation and duplicity. She tells readers a partial tale of her youth, which is both biased and incomplete. Some events in her past are concealed, or glossed over. Through a deft handling of rhetoric, she truly makes her life story, written for her friend the Marquise, a literary act of seduction. As a result, an ambiguous relationship develops between the reader and the narrating heroine, which keeps oscillating between secret understanding and critical distance.
\end{abstract}

Keywords (En): simulation ; dissimulation ; duplicity ; novel ; Marivaux

Mots-clés (Fr): simulation ; dissimulation ; duplicité ; roman ; Marivaux

\section{Introduction}

S'il est une œuvre dans laquelle simulation et dissimulation sont présentes, c'est bien La Vie de Marianne, non au niveau lexical (on trouve seulement deux occurrences du substantif « dissimulation ${ }^{2} »$, et aucune de « simulation »), mais au niveau diégétique et narratif. Ce dernier roman de Marivaux, rédigé entre 1726 et 1741 et publié entre 1731 et 1742 , est en lui-même une simulation. D'une part, l'auteur invente une voix de femme, et qui plus est, cette voix est double : celle d'une jeune fille de quinze ans, Marianne, et celle du même personnage devenu Comtesse de ${ }^{* * * 3}$ et narratrice de sa propre histoire, à l'âge de cinquante ans environ. D'autre part, selon un procédé courant au dix-huitième siècle, Marivaux simule l'authenticité du manuscrit. Dans un premier temps, il reproduit l'Avertissement d'un éditeur qui met en place la fiction du non fictif, en

\footnotetext{
1 Toutes les références à La Vie de Marianne, ainsi que les citations, proviennent de l'édition de Jean Marie Goulemot, Le Livre de Poche « Les Classiques de Poche », n²1032, Paris, 2007, 2014.

2 - «C'était un Amant que je voyais ; il se montrait à visage découvert, et je ne pouvais, avec mes petites dissimulations, parer l'évidence de son amour. » (p. 131)

- «Ce sera lui manquer de reconnaissance, et je ne saurais me résoudre à une dissimulation si ingrate » (p. 351). Marianne ne peut se résoudre ici à duper sa bienfaitrice Mme de Miran. Elle lui avoue que Mme de Fare a appris, par la servante Favier, qu'elle n'était pas la fille d'amis de Mme de Miran, mais une simple orpheline. Le comportement de Mme de Fare a alors changé : « ses manières étaient bien changées, ma mère, je suis obligée de vous l'avouer ; je croirais faire une perfidie si je vous le cachais. » (p. 354).

${ }^{3}$ Le titre complet, La Vie de Marianne ou les Aventures de Madame la Comtesse de ***, révèle la promotion sociale inattendue de Marianne l'enfant trouvé.
} 
prétendant que le récit qui va suivre n'est pas «une histoire simplement imaginée » (p. 55). Puis il y ajoute un préambule ${ }^{4}$ attribué à un ami de l'éditeur : celui-ci est censé avoir découvert un manuscrit authentique vieux de quarante ans, écrit par une femme qui raconterait sa vie par lettres à l'une de ses amies. En écho, par la suite, le romancier fait régulièrement prétendre à la narratrice elle-même que l'histoire qu'elle raconte n'a rien d'un roman ${ }^{5}$.

Dans La Vie de Marianne, Marivaux exploite toutes les ressources d'un procédé que Jean ROUSSET a présenté comme le principe structurant de toute son œuvre, romanesque comme théâtrale : le « double registre » (1962: 45-64). Dans de nombreuses pièces de Marivaux, les héros agissants sont regardés par des personnages spectateurs, et dans les deux derniers romans de Marivaux, Le Paysan parvenu et La Vie de Marianne, on retrouve ce dédoublement entre un regardant, qui est le narrateur dans le présent de l'écriture, et un regardé, lui-même qui s'observe et se décrit comme personnage dans le passé. À la différence des pièces de théâtre, dans ces deux romans à la première personne, «le personnage agissant et le personnage spectateur n'en font plus qu'un » (ROUSSET, 1962: 51)

Devenue narratrice de sa propre histoire, la Comtesse considère de loin, à distance, la jeune fille qu'elle a été, avec davantage de lucidité et maturité. Mais son regard est fortement teinté de partialité et de duplicité. Comme dans le passé la jeune coquette Marianne savait simuler et dissimuler pour séduire, la narratrice fait tout pour séduire sa narrataire, l'amie Marquise à qui elle destine ses lettres, dans le présent de l'écriture. Le regretté critique René DÉMORIS ${ }^{6}$ a signalé chez les narrateurs de Marivaux cette permanence du désir de séduire, qui change seulement de moyens : «[...] Les moyens seuls de la séduction ont changé : le corps a laissé place à l'écriture. C'est donc bien dans une entreprise littéraire que s'est engagé le narrateur. » (1975: 412-413).

Il est donc nécessaire d'analyser les formes et les effets de la simulation et de la dissimulation chez l'héroïne, mais peut-être plus encore chez la narratrice de $L a$ Vie de Marianne.

Au niveau diégétique, la jeune Marianne est une simulatrice qui se transforme souvent en actrice sur la scène du monde. Elle use de coquetterie, manipule certains de ses interlocuteurs et s'abandonne à l'affabulation romanesque quand elle s'invente des origines aristocratiques. En parfaite continuité avec cette tendance initiale, la narratrice autodiégétique fait preuve de dissimulation et de duplicité. Elle livre un récit partiel et partial de sa jeunesse : certains éléments de son passé sont occultés, ou racontés de manière tendancieuse. Le récit de sa vie, destiné à son amie Marquise, devient une véritable entreprise littéraire de séduction, grâce à une rhétorique savamment maîtrisée. Il en découle une relation ambiguë entre l'hérö̈ne-narratrice et le lecteur virtuel, celui que postule tout

\footnotetext{
${ }^{4}$ p. 57-58.

5 Voir par exemple : «Ce début paraît annoncer un roman: ce n'en est pourtant pas un que je raconte ; je dis la vérité comme je l'ai apprise de ceux qui m'ont élevée » (p. 60), ou : «C'est qu'au lieu d'une histoire véritable, vous avez cru lire un roman. Vous avez oublié que c'était ma vie que je vous racontais $[\ldots] \gg($ p. 457$)$.

${ }^{6}$ Ce spécialiste de la peinture et de la littérature des XVII ${ }^{\text {m̀e }}$ et XVIII ${ }^{\text {ème }}$ siècles est décédé récemment, en janvier 2016.
} 
texte : le lecteur est partagé entre la complicité et la distance critique envers l'héroïne-narratrice.

\section{La simulation chez l'hérö̈ne}

La simulation est une attitude fréquente chez la jeune Marianne. Elle se révèle d'emblée une coquette et une actrice douée sur la scène du monde. Marianne a de véritables prédispositions pour la mise en scène de soi. Dès son arrivée à Paris, la jeune fille oublie le naturel et s'adonne intuitivement à la coquetterie, ce qui lui procure une forte jouissance narcissique. C'est notamment le cas lors de la scène bien connue dans l'église, où elle se révèle très douée pour attirer les regards. En effet, elle sait exploiter avec talent la hauteur des tableaux pour montrer la beauté de ses yeux, ou remettre savamment en place sa coiffe afin de pouvoir exhiber la finesse de sa main ou le galbe de son bras ${ }^{7}$.

Avant tout, le récit fait régulièrement sentir, à défaut de l'avouer toujours explicitement, l'hypocrisie et la mauvaise foi de la jeune fille. Le lexique du jeu théâtral est très présent. Par exemple, après l'examen de son entorse par un chirurgien, lorsque Valville veut la reconduire chez elle, on apprend que la jeune fille trouve un «expédient» (p. 130) - ce terme suggère bien l'artifice et la feinte - pour ménager sa vanité et lui permettre de ne pas révéler qu'elle loge chez une lingère. Elle prétend d'abord vouloir se retirer seule. Puis, quand Valville insiste pour la reconduire lui-même, elle se met à pleurer, ce qui lui réussit bien plus que toute autre attitude, en lui donnant un «air de dignité romanesque » (p. 140) qui dispose le jeune homme en sa faveur. Et c'est encore grâce au bénéfice qu'elle retire de ses larmes - qu'on peut juger en partie artificielles qu'elle parvient à surmonter son humiliation, au moment même où elle doit se résoudre à avouer à Valville qu'elle vit chez une lingère ${ }^{8}$. D'ailleurs, les larmes de Marianne coulent à flots au cours du récit ${ }^{9}$, notamment lors de sa comparution devant un tribunal de famille chez le Président, dans la Septième Partie. La jeune fille pleure tellement qu'on peut souvent la soupçonner de simuler ou de surjouer son chagrin, afin d'apitoyer ses interlocuteurs. La Comtesse suggère d'ailleurs la dimension théâtrale de cette posture lacrymale de Marianne : «je pleurais moins par chagrin, je pense, que par mignardise. » (p. 499).

En outre, Marianne sait jouer sur l'air' et le ton, comme une actrice. Dans un premier temps, à son arrivée à Paris, la jeune orpheline qui a besoin de soutien

\footnotetext{
${ }^{7}$ Une telle maîtrise chez la jeune fille de l'art de voiler, puis de dévoiler progressivement ses atouts, pourrait même être perçue comme l'annonce métaphorique de la maîtrise du récit par la Comtesse, et du fonctionnement même de ce récit. Comme Marianne à l'église, la narratrice sait voiler certains éléments de sa vie et les dévoiler peu à peu, en adoptant une rhétorique de la dissimulation, comme on le verra plus tard.

8 « Je pleurai donc, et il n'y avait peut-être pas de meilleur expédient pour me tirer d'affaire, que de pleurer et de laisser tout là. Notre âme sait bien ce qu'elle fait, ou du moins son instinct le sait bien pour elle. » (p. 139).

${ }^{9}$ Voir par exemple : «Un torrent de pleurs termina mon discours » (p. 414).

${ }^{10}$ On relève plus de deux cents occurrences du substantif «air » et une centaine du substantif «ton » dans La Vie de Marianne : c'est très significatif.
} 
accepte le manège hypocrite du faux dévot. Elle use alors avec virtuosité de l'art et de l'air de la feinte :

Ce discours était assez net, et il était difficile de parler plus français : je fis semblant d'être distraite pour me dispenser d'y répondre ; mais un baiser qu'il m'appuyait sur l'oreille en me parlant, s'attirait mon attention malgré que j'en eusse, et il n'y avait pas moyen d'être sourde à cela ; aussi ne le fus-je pas. Monsieur, ne vous ai-je pas fait mal ? m'écriai-je d'un air naturel, en feignant de prendre le baiser qu'il m'avait donné pour le choc de sa tête avec la mienne. Dans le temps que je disais cela, je descendais de carrosse, et je crois qu'il fut la dupe de ma petite finesse, car il me répondit très naturellement que non. (p. 96)

L'abondance du lexique de la simulation démontre ici le réel talent de la jeune fille pour l'hypocrisie. Il en va de même quand elle vient de rencontrer Valville. Par bienséance, elle veut lui cacher son attirance. Mais paradoxalement, parce qu'elle est amoureuse, elle cherche aussi à la lui faire sentir. Elle use alors, encore une fois, d'une rhétorique corporelle bien maîtrisée :

Quoi ! partir si tôt ? me dit-il en jetant sur moi le plus doux de tous les regards. Il le faut bien, repris-je en baissant les yeux d'un air triste. (p. 131)

Et c'est aussi avec un art consommé de la séduction que Marianne sait varier les tons. Elle adopte un «ton ingénu ${ }^{11} »($ p. 72$)$ pour jouer la comédie de la naïveté auprès de M. de Climal quand il revient la voir chez la Dutour. Pourtant, comme le lecteur l'a appris plus tôt ${ }^{12}$, son point de vue sur Climal a alors définitivement changé. Sous l'influence de son amour naissant pour Valville, et depuis qu'elle a vu les réactions de Climal quand il a surpris Valville à ses genoux, elle ne se fait plus aucune illusion sur ce Tartuffe envers qui elle n'éprouve plus que répulsion. De même, quand elle fait le récit de ses mésaventures à Mademoiselle Varthon, Marianne ne peut s'empêcher de l'orner d'un ton pathétique propre à apitoyer son interlocutrice ${ }^{13}$.

Enfin, Marianne apparaît comme une jeune fille calculatrice, qui sait résoudre à son avantage tous ses cas de conscience. Elle se livre souvent à de longues introspections casuistes, afin de dissiper ses scrupules. Ainsi, c'est au terme d'un raisonnement très tendancieux que la jeune coquette s'autorise à accepter le beau linge offert par M. de Climal :

\footnotetext{
${ }^{11}$ Voir aussi : «Ah ! ma mère, lui repartis-je d'un ton pénétré, quelles délices pour moi ! (p. 424).

12 « Trois jours auparavant, en découvrant qu'il m'aimait, je m'étais contentée de penser que c'était un hypocrite, que je n'avais qu'à laisser être ce qu'il voudrait, et qui n'y gagnerait rien ; mais à présent je n'en restais pas là, je ne me contenais plus pour lui dans cette tranquille indifférence. Ses sentiments me scandalisaient, m'indignaient; le cœur m'en soulevait. En un mot, ce n'était plus le même homme à mes yeux : les tendresses du neveu, jeune, aimable et galant, m'avaient appris à voir l'oncle tel qu'il était, et tel qu'il méritait d'être vu; elles l'avaient flétri, et m'éclairaient sur son âge, sur ses rides, et sur toute la laideur de son caractère. » (p. 170).

13 «Mon récit devient intéressant; je le fis, de la meilleure foi du monde, dans un goût aussi noble que tragique ; je parlai en déplorable victime du sort, en héroïne de roman, qui ne disait pourtant rien que de vrai, mais qui ornait la vérité de tout ce qui pouvait la rendre touchante, et me rendre moimême une infortunée respectable. » (p. 435).
} 
Je consultais donc en moi-même ce que j'avais à faire ; et à présent que j'y pense, je crois que je ne consultais que pour perdre du temps. [...] Par là je reculais une rupture avec M. de Climal, et je gardais ce qu'il me donnait. [...] Ce petit cas de conscience ainsi décidé, mes scrupules se dissipèrent et le linge et l'habit me parurent de bonne prise. (p. 94-95).

Et quand elle se décide enfin à le lui rendre, elle parvient à conserver malgré tout la robe, après un débat intérieur hypocrite et un prétendu raisonnement qui est en vérité empreint d'une mauvaise foi certaine : «Je la gardai donc, et sans scrupule, j'y étais autorisée par la raison même : l'art imperceptible de mes raisonnements m'avait conduit jusque-là, et je repris courage jusqu'à nouvel ordre. » (p. 198). Dans le domaine amoureux, Marianne use d'une casuistique tout aussi tendancieuse, ce qui confirme son aptitude à la simulation et la dissimulation. Chez Valville, après l'incident qui provoque son entorse, elle met en place une stratégie pleine de ruse. Elle refuse d'abord de montrer sa cheville au chirurgien, puis elle finit par accepter de le faire, car elle calcule qu'elle en aura tout le bénéfice: Valville pensera qu'elle a d'abord refusé par pudeur et par modestie, et quand le chirurgien l'exigera, elle pourra alors exhiber devant le jeune homme son «joli petit pied» (p. 125) sans paraître effrontée. L'examen de conscience de Marianne se conclut d'ailleurs par un discours sentencieux qui légitime sa propre mauvaise foi, en en faisant un trait de caractère universel : «Combien dans le monde y a-t-il d'honnêtes gens qui me ressemblent, et qui, pour pouvoir garder une chose qu'ils aiment, ne fondent pas mieux leur droit d'en jouir que je faisais du mien dans cette occasion-là. » (p. 125-126). Un peu plus tard, la jeune fille, opportunément vêtue d'un négligé qui met en avant ses charmes naturels, adresse à Valville, au parloir du couvent, un long discours émouvant pour tenter de le détourner de l'aimer. Elle sait sans doute qu'elle va obtenir l'effet inverse, et c'est bien ce qui se produit : à l'issue de ce discours, Valville devient plus amoureux que jamais, et Madame de Miran consent à la relation entre son fils et Marianne. Mais Madame de Miran, elle, n'est pas dupe du manège de la jeune fille, puisqu'elle s'exclame avec une grande lucidité : «Quelle dangereuse petite fille tu es, Marianne. » (p. 267), et elle dira en écho, quelques pages plus loin : «Ah! la bonne petite hypocrite ! (p. 306).

Si Marianne simule auprès de son entourage, elle s'invente aussi une vie, en se transformant en romancière de sa propre existence. René DÉMORIS a défini avec justesse ce qu'il appelle la «fonction fabulatrice» qu'il voit à l'œuvre tout particulièrement dans les romans de Marivaux :

C'est toute sa vie, et pas seulement à son terme, que l'être se raconte à lui-même une histoire qui n'est peut-être pas vraie, mais qui présente l'avantage d'être à la fois agréable et utile : n'est-ce pas la prétention du roman ? [...] Le personnage se trouve devant sa vie dans une position assez analogue à celle que connaît l'écrivain en train de bâtir une fiction. (1975 : 407-408)

Dans La Vie de Marianne, non seulement l'héroïne simule et dissimule, mais on peut dire aussi qu'elle fabule et affabule : elle se sert de son imagination pour s'inventer une origine fantasmatique. Elle se raconte à elle-même une belle histoire, celle d'une princesse abandonnée, d'après le récit qui lui a été fait des 


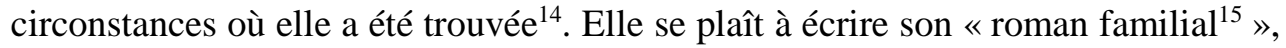
en mettant l'accent sur ses prétendues origines nobles qu'elle ne cesse de rappeler à ses multiples interlocuteurs ${ }^{16}$. C'est ainsi qu'elle déclare à M. de Climal, tristement et en versant quelques larmes : «Hélas ! Monsieur, [...], quoique je n'aie rien, et que je ne sache à qui je suis, il me semble que j'aimerais mieux mourir que d'être chez quelqu'un en qualité de domestique; et si j'avais mon père et ma mère, il y a toute apparence que j'en aurais moi-même, au lieu d'en servir à personne. » (p. 87) Elle va même jusqu'à attribuer à son «sang » la délicatesse de ses manières (p. 86), et elle perçoit un indice incontestable de son rang élevé dans sa capacité innée à se laisser habiller par une femme de chambre ${ }^{17}$.

Par bien des aspects, Marianne est donc une jeune fille simulatrice, qui ment aux autres ou se ment à elle-même, en utilisant toutes les ressources de la séduction physique ou verbale et en s'inventant des origines nobles. Mais le triomphe ultime de l'héroïne consiste à passer du statut d'acteur à celui d'auteur ${ }^{18}$. Marianne devenue narratrice transforme la séduction textile de sa jeunesse en séduction textuelle, grâce à sa maitrise du récit.

\section{Dissimulation et duplicité chez la narratrice}

En totale contradiction avec le naturel et l'authenticité dont elle prétend pourtant régulièrement être la parfaite illustration ${ }^{19}$, la narratrice dissimule certains éléments de sa vie à sa destinataire, transformant ainsi ce qu'elle raconte en un récit partiel.

Les ellipses, zones d'ombre et lacunes sont nombreuses. D'abord, et cela n'a rien d'anodin dans la société d'Ancien Régime où le nom est un marqueur de l'appartenance sociale et un gage de l'être, la narratrice choisit de dissimuler sa véritable identité. Celui qui est censé avoir découvert son manuscrit constate : « nous ne savons qui elle était. » (p. 58). Et la narratrice elle-même signale que sa narrataire, son amie la Marquise, sera la seule détentrice du secret de son identité : « N'oubliez pas que vous m'avez promis de ne jamais dire qui je suis; je ne veux être connue que de vous. » (p. 60). Certes, le titre complet du roman, quand il n'est pas tronqué par les éditeurs, permet de connaître son nouveau rang social, mais

\footnotetext{
14 « Remarquez qu'entre les personnes qui avaient été tuées, il y avait deux femmes : l'une belle et d'environ vingt ans, et l'autre habillée comme le serait une femme de chambre.

Si l'une des deux était ma mère, il y avait plus d'apparence que c'était la jeune et la mieux mise, parce que je lui ressemblais un peu, du moins à ce que disaient ceux qui la virent morte, et qui me virent aussi, et que j'étais vêtue d'une manière trop distinguée pour n'être que la fille d'une femme de chambre. » (p. 61).

${ }^{15}$ Voir Marthe ROBERT, Roman des origines et origines du roman, Grasset, 1972.

${ }^{16}$ Voir l'ouvrage, même s'il est un peu daté et trop systématique, d'Annick Jugan, Les variations du récit dans La Vie de Marianne de Marivaux.

${ }^{17}$ «Il fallait bien soutenir mon rang, et c'était là de ces choses que je saisissais on ne peut pas plus vite ; j'avais un goût naturel, ou, si vous voulez, je ne sais quelle vanité délicate qui me les apprenait tout d'un coup, et ma femme de chambre ne me sentit point novice» (p. 331).

${ }^{18}$ L'origine étymologique des deux termes est peut-être le verbe ago, agere en latin : faire, agir. L'acteur est celui qui 'fait' une pièce et l'auteur celui qui pousse à agir, le fondateur, le créateur.

19 « car où voulez-vous que je prenne un style ? [...] Au reste, je parlais tout à l'heure de style, je ne sais pas seulement ce que c'est. » (p. 58, 60).
} 
son nouveau nom est dissimulé sous les astérisques : la Comtesse de ***. Enfin, en un jeu spéculaire aussi intrigant qu'irritant, on n'en sait pas plus sur l'identité de la destinataire du récit : on apprendra seulement au détour d'une page qu'elle est Marquise (p. 457). De la même façon, on ignore quasiment tout de ce qui se passe avant les quinze ans de Marianne. Après avoir raconté de seconde main (inévitablement) l'attaque du carrosse, événement traumatique fondateur qui scelle le mystère de son origine (p. 60-63), la narratrice passe sous silence la période de l'enfance où elle vit chez un Curé et sa sœur ${ }^{20}$. Son récit rétrospectif s'intéresse avant tout à l'arrivée de Marianne à Paris : elle décrit donc seulement les premiers mois et émois de la jeune fille. Selon un principe de composition binaire, ou de réversibilité des contraires, et de manière très 'romanesque', l'héroïne va alors connaître en quelques mois une succession de joies et de succès intenses, mais aussi de fortes désillusions. Comme dans le théâtre de Marivaux, une expérience d'abord heureuse se transforme souvent en son contraire, une douloureuse épreuve intérieure. Ce récit mouvementé des premières aventures de Marianne occupe les huit premières parties. Mais il s'interrompt brutalement à la fin de la Huitième Partie, au moment même où la narratrice vient d'annoncer avec malice : «Je suis née pour avoir des aventures, et mon étoile ne m'en laissera pas manquer.» (p. 503) Paradoxalement, c'est alors l'histoire enchâssée de Tervire qui occupe les trois dernières parties du récit de la Comtesse. En effet, au couvent, Marianne annonce à son amie Tervire qu'elle veut devenir définitivement religieuse, et Tervire tente alors de l'en dissuader en lui racontant sa propre histoire. Au récit de vie de Marianne succède donc celui de Tervire, non sans invraisemblance, car la longue histoire de Tervire est censée être un discours rapporté. Même si les liens entre les deux récits de vie sont étroits ${ }^{21}$, le lecteur est donc confronté à une béance narrative : il ne connaîtra jamais la suite et la fin du parcours social de Marianne. Enfin, rien n'est indiqué sur le lieu et l'époque de l'écriture : «le lieu de l'écriture reste enveloppé dans le même mystère que le lieu de la naissance ${ }^{22}$. ». D'ailleurs, bien rares sont au cours du récit les indices topographiques précis ${ }^{23}$. Et le lecteur ne saura jamais comment Marianne l'orpheline acquiert son titre de noblesse, ce qui laisse libre cours à toutes les hypothèses et maintient l'œuvre ouverte. Devientelle Comtesse en retrouvant le reste de sa famille, ou bien est-ce grâce à son mariage avec Valville, ou avec un autre noble ? L'inachèvement du récit entraîne un ultime repli de la narratrice sur le secret de ses origines.

La narratrice dissimule donc beaucoup, mais plus encore elle fait preuve de duplicité et de mauvaise foi, en confiant à sa narrataire un récit partial de ses aventures. En effet, régulièrement, la Comtesse s'efforce de justifier le comportement de la jeune fille qu'elle a été. Lorsqu'elle retrace la scène où Madame Dutour, vexée que sa boutique n'ait pas eu les faveurs de M. de Climal, reproche à Marianne d'avoir accepté un linge trop beau pour être honnête, la narratrice se montre solidaire de la jeune fille coquette qu'elle était. La voix de

\footnotetext{
20 « Je passe tout le temps de mon éducation dans mon bas âge, pendant lequel j'appris à faire je ne sais combien de petites nippes de femme, industrie qui m'a bien servi dans la suite. » (p. 63).

${ }^{21}$ Voir notamment l'Introduction de Jean Marie Goulemot, p. 16-17.

${ }^{22}$ DIDIER, $1987: 100$.

${ }^{23}$ Voir GuiLhemBet, 2014b : 277-282.
} 
Marianne et celle de la narratrice se confondent pour dissocier la faute dont elle est accusée de la «pureté de [s]es intentions » (p. 100). Une telle attitude, très casuiste, permet d'inciter à l'indulgence la narrataire, et par extension tous les lecteurs. Le récit vise d'ailleurs souvent à les persuader que même si l'on peut juger immorale l'attitude de Marianne, cette dernière doit bénéficier de circonstances atténuantes : «Mon raisonnement était sans doute une erreur, mais non pas un crime. [...] Et voyez quelle ressource que le vice des hommes ! N'était-ce pas là de quoi renverser une cervelle aussi jeune que la mienne ?» (p. 101). Si la narratrice emploie ici une interrogation rhétorique, c'est bien pour atténuer la responsabilité de la jeune fille qu'elle a été ${ }^{24}$ et pour prendre sa destinataire au piège d'une rhétorique parfaitement maîtrisée.

Enfin, la narratrice, simulatrice douée, feint constamment de s'adresser à sa destinataire, ce qui participe d'une stratégie rhétorique de séduction. La Comtesse insère, le plus souvent au début ou à la fin d'une partie, un dialogue imaginaire, fictif, simulé, avec son amie : «je m'imagine que je vous parle, et tout passe dans la conversation : continuons-la donc. » (p. 90). Elle donne à son récit par lettres l'allure d'une authentique conversation, en employant tour à tour le pronom « vous », parfois associé à une tournure hypocoristique comme « ma chère amie » (p. 58 et 115), et le pronom première personne du pluriel, afin de créer l'illusion d'une forte connivence entre femmes : «Nous verrons qui c'était dans la suite [...]» (p. 163), «Mais achevons d'écouter Mme de Miran qui continue [...]» (p. 242). De surcroît, avec une grande habileté, elle imagine et anticipe les critiques ou objections de sa lectrice. Elle feint d'abord de croire que le récit de sa vie pourrait ennuyer la Marquise : «Le commencement de ma vie contient peu d'événements, et tout cela aurait bien pu vous ennuyer.» (p. 116). Toutefois, l'emploi du conditionnel suggère déjà que cet ennui présumé ne peut exister. Et dès la phrase suivante, tout au contraire, elle signale l'intérêt de son amie : «Vous me dites que non, vous me pressez de continuer, je vous en rends grâce, et je continue [...] » (p.116). Enfin, elle sollicite le pardon de sa destinataire pour toutes les digressions qu'elle prétend ne pas pouvoir éviter : «Mais je m'écarte toujours; je vous en demande pardon, cela me réjouit ou me délasse ; et encore une fois, je vous entretiens. » (p. 107) ${ }^{25}$.

À cette simulation d'un dialogue s'ajoute la dissimulation, autre ruse rhétorique pour maintenir et intensifier la curiosité de la Marquise. Dans une correspondance qui s'effectue par envois successifs et irréguliers, la narratrice cache à sa narrataire le moment précis où elle va recevoir la suite de ses aventures. Et si elle lui annonce sans cesse l'histoire de Tervire, elle diffère toujours le moment de la lui raconter ${ }^{26}$. En outre, pour tenir en haleine son amie, la narratrice

\footnotetext{
${ }^{24}$ Cf le narrateur du Paysan Parvenu: «N'était-ce pas là la pomme d'Adam tout revenue pour moi ? » (GF Flammarion n ${ }^{\circ} 1437$, Paris, 2010, éd. Erik Leborgne, p. 26). Dans La Vie de Marianne, on retrouve régulièrement la dissociation casuiste entre l'intention et les actes (par exemple, p. 413). ${ }^{25} \mathrm{Cf}$ « Cette réflexion a coulé de ma plume sans que j’y prisse garde ; heureusement elle est courte, et j'espère qu'elle ne vous ennuiera pas » (p. 276).

${ }^{26}$ Alors qu'elle devait être intégrée à la Cinquième partie, elle est promise pour le début de la Sixième partie, mais encore remise à plus tard, à tel point que la narrataire est censée exprimer son impatience, dans le discours fictif que lui prête la narratrice : "Mais l'histoire de cette Religieuse
} 
utilise régulièrement des prolepses narratives, comme «laissez-moi faire, je ne serai pas toujours chez Madame Dutour. » (p. 116) ${ }^{27}$. Ces prolepses ne révèlent que très partiellement les faits à venir : elles servent donc avant tout à attiser la curiosité de la narrataire.

Ainsi, force est de constater que la jeune coquette simulatrice et dissimulatrice s'est convertie en une narratrice retorse qui se livre à une entreprise de séduction textuelle. Le lecteur virtuel, celui que postule tout récit, se laisse-t-il alors pleinement séduire par ce récit tellement maîtrisé, au risque d'une perte totale de sa lucidité ?

\section{La relation ambiguë entre le lecteur et l'hérö̈ne-narratrice}

Le lecteur (ou la lectrice, bien entendu) éprouve inévitablement un sentiment de complicité avec l'héroïne-narratrice. En effet, il est censé lire un texte intime, privé, une correspondance fictive entre deux femmes amies. Il entre donc, bien malgré lui et comme par effraction, dans un récit qui ne lui est pas destiné et qui lui propose - ou même lui impose - un point de vue unique, celui d'une narratrice autodiégétique toute-puissante, Marianne qui raconte l'histoire de Marianne. Pris au piège de ce récit tendancieux à la première personne dont il devient lui aussi le destinataire, le lecteur est sans doute tout aussi impatient que la Marquise ${ }^{28}$ de connaître la suite des aventures de Marianne. La Marquise est un substitut fictif du lecteur réel : elle est « déléguée par l'auteur pour figurer le lecteur, dont elle assure la présence au cœur de l'œuvre.»(DIDIER, 1987 : 15). Et Jean Marie Goulemot met l'accent sur l'importance décisive de ce personnage pour orienter le jugement du lecteur : «À travers les réactions que Marianne prête à sa correspondante se construit un imaginaire du lecteur.» (p. 14). Le lecteur séduit, voire hypnotisé, peut légitimement éprouver un sentiment de forte complicité avec Marianne la jeune coquette envers qui il ressentira une indulgence amusée, comme la Comtesse et la Marquise elles-mêmes. Il est souvent partagé entre l'admiration et la compassion envers Marianne. En effet, le récit de la Comtesse met en avant la dignité de la jeune fille face à tous les obstacles qu'elle rencontre ${ }^{29}$, ce qui incite le lecteur à accorder toute son estime à Marianne, présentée comme la victime courageuse d'une société qui la rejette, notamment quand elle est confrontée à un conseil de famille qui n'éprouve d'abord aucune indulgence à son égard ${ }^{30}$. Mais le récit joue aussi sur le pathos quand il dramatise certains moments douloureux, par

\footnotetext{
que vous m'avez tant de fois promise, quand viendra-t-elle ? me dites-vous. » (p. 453). Et ce n'est que dans la Neuvième Partie qu'elle débutera vraiment.

27 Voir aussi «Nous verrons qui c'était dans la suite; c'est ici que mes aventures vont devenir nombreuses et intéressantes : je n'ai pas encore deux jours à demeurer chez Madame Dutour » (p. 163), ou «(il ne me disait de si jolies choses qu'à cause qu'il commençait à n'en plus sentir de si tendres) » (p. 427).

${ }^{28}$ Voir par exemple : «Dites-moi, ma chère amie, ne serait-ce point un peu par compliment que vous paraissez si curieuse de voir la suite de mon histoire? » (p. 115) ou «Vous me demandez quand viendra la suite de mon Histoire ; vous me pressez de vous l'envoyer. Hâtez-vous, me dites-vous, je l'attends [...] » (p. 457).

${ }^{29}$ Marianne est décrite comme une « belle âme » à deux reprises (p. 245, 256).

${ }^{30}$ Chez le Ministre parent de Madame de Miran (Sixième et Septième parties).
} 
exemple lorsque Marianne, orpheline toujours en quête de la tendresse d'une mère, est la proie du dévot libertin M. de Climal, ou quand elle est la cible de critiques acerbes de la part de certains parents de Madame de Miran. Le lecteur est alors ému par le sort tragique de Marianne qui ne peut le laisser indifférent. De surcroît, comme on l'a vu, l'emploi de la première personne favorise l'adhésion du lecteur au récit de la Comtesse ${ }^{31}$ : tout l'univers du récit existe seulement grâce à elle, elle est à la fois acteur et auteur, spectatrice et juge de Marianne, et son regard surplombant est inévitablement biaisé. Le lecteur peut éprouver un sentiment de forte proximité et de complicité avec une narratrice qui organise sa narration de manière à séduire sa narrataire, et donc par extension tous les lecteurs.

Toutefois, à la compassion envers une hérö̈ne vulnérable et à la complicité avec une narratrice rouée, vient se superposer une certaine distance critique. En effet, le lecteur qui n'est pas totalement naïf prend bien vite conscience de la grande faculté de simulation et de dissimulation que partagent l'héroïne et la narratrice. En définitive, ce que propose Marivaux dans La Vie de Marianne, c'est donc un nouveau mode de lecture du roman, fondé sur une plus grande lucidité du lecteur, plutôt que sur son adhésion naïve à un récit qui se révèle partiel et partial. Implicitement, le lecteur est invité à conserver une certaine défiance envers les propos tendancieux d'une narratrice qui raconte l'histoire de sa propre vie pour séduire ou apitoyer sa narrataire. Encore une fois, il faut s'appuyer sur les analyses éclairantes de René DÉMORIS :

Le langage du narrateur est partiel et partial, fût-ce par omission, et c'est à son croisement avec celui du personnage agissant que le lecteur est convié à rechercher la vérité. [...] À la différence de ce qui se passait pour les héros des mémoires antérieurs, qui conjuraient bien loin le spectre maudit du mensonge romanesque, Marivaux ne cache pas que l'ultime bonheur de son personnage - et sa ressource légitime - a été de devenir son propre romancier. $(1975: 412,413)$

Le critique donne ici au lecteur un rôle déterminant, celui de découvrir «la vérité » du récit, qui se situe dans un rapport dialectique entre authenticité et simulation, sincérité et dissimulation. C'est bien Marivaux lui-même qui met son lecteur sur la voie, en faisant de sa narratrice une romancière qui transforme sa vie en roman. Comme le récit est peu fiable, le lecteur est incité par l'auteur à conserver toute sa lucidité, afin de ne pas se laisser prendre au piège de la rhétorique bien maîtrisée et parfois perverse de la narratrice. N'est-ce pas là un signe éclatant de la modernité de Marivaux?

\footnotetext{
31 «Grâce à la première personne qui unit étroitement le narrateur et le personnage narré, tout le roman bascule donc pour s'ordonner autour d'un moi central et dominateur, à la fois tout-puissant puisqu'il soumet le monde à sa propre expérience, et pourtant limité dans sa vision, puisqu'il doit renoncer à l'omniscience de l'auteur pour se borner à sa seule perspective de narrateur inclus dans l'univers qu'il raconte. Ce perspectivisme intériorise le roman en le plaçant tout entier sous le regard de ce narrateur qui le réfléchit en lui-même. » (RousseT, 1973 : 112).
} 


\section{Conclusion}

Ainsi, dans La Vie de Marianne, simulation et dissimulation sont des stratégies très présentes et communes à l'héroïne et à la narratrice. Telle une actrice, la jeune coquette simulatrice se sert de son éloquence corporelle ou verbale pour plaire à autrui, et elle se raconte à elle-même une histoire. Quand elle est plus âgée, elle utilise non plus son corps, mais l'écriture, pour procéder à une entreprise littéraire de séduction fondée sur la dissimulation, en devenant romancière de sa propre existence. La narratrice ne dit pas tout à sa destinataire, et elle tente de la prendre au piège d'une rhétorique savante.

Face à un tel récit, le lecteur est implicitement invité par le romancier à naviguer entre l'adhésion émue et complice et la distance critique envers une héroïne-narratrice manipulatrice. L'autre grand roman à la première personne de Marivaux, Le Paysan parvenu (1734-35), visait déjà à susciter une réaction du même ordre chez le lecteur, tiraillé entre la complicité indulgente envers le jeune Jacob et la distance envers le récit du narrateur, Jacob devenu lui aussi auteur de ses Mémoires et aristocrate anonyme, M. ***.

\section{BIBLIOGRAPHIE}

COUlet Henri (1975), Marivaux romancier. Essai sur l'esprit et le cœur dans les romans de Marivaux, Paris, Armand Colin.

DÉMORIS René (1975), Le roman à la première personne, du classicisme aux Lumières, Paris, Armand Colin, p. 397-413.

DENEYS-TUNNEY Anne (1992), « Marianne ou le babillage du corps », in : Écritures du corps. De Descartes à Laclos, Paris, PUF, «Écriture », p. 71-129.

DIDIER Béatrice (1987), La Voix de Marianne. Essai sur Marivaux, Paris, José Corti.

GenetTe Gérard (1982), «Fins de Marianne, fins de Jacob », in : Palimpsestes, Paris, Seuil, « Poétique », p. 185-191.

GuilHEMBET Jacques (2014a), «Artifice et belles dentelles : de la séduction du textile à la séduction textuelle dans La Vie de Marianne ", in : Revue de littératures française et comparée "Méthode! », n²4 (Agrégation de Lettres 2015), Bandol, éd. de Vallongues, p. 147-154.

GuILHEMBET Jacques (2014b), L'Euvre romanesque de Marivaux. Le parti pris du concret, Classiques Garnier, «L'Europe des Lumières » (33), Paris, 2014.

GuILHEMBET Jacques (2015), «De la séduction textile à la séduction textuelle dans Le Paysan parvenu et La Vie de Marianne de Marivaux », in : Criação \& Crítica, $\mathrm{n}^{\circ} 15$, p. 55-73, http://revistas.usp.br/criacaoecritica.

GuILHEMBET Jacques (2015), «Enjeux du costume et de la parure dans l'œuvre romanesque de Marivaux », in : Sociopoétique du textile à l'âge classique. Du vêtement et de sa représentation à la poétique du texte, Paris, Hermann, p. 401-421 [Actes du colloque international «Sociopoétique du costume: costumes, habits et accessoires, entre vie sociale et fiction, dans la France de 
l'âge classique (XVII ${ }^{\text {ème}}-X V I I I{ }^{\text {ème }}$ siècle) », Centre National du Costume de Scène (Moulins), 4-6 septembre 2014].

GUILHEMBET Jacques (2015), «Stupeur, rougeur et tremblements de l'hérö̈ne dans La Vie de Marianne », in : Malice $\mathrm{n}^{\circ}$ 5, publication en ligne du CIELAM, http://cielam.univ-amu.fr/publication/1416 [Actes du Colloque international «Marivaux entre les genres. Le corps, la parole, l'intrigue » (16 et 17 janvier 2015), Université d'Aix-Marseille, UFR ALLSH-CIELAM, Aix-en-Provence].

JUGAN Annick (1978), Les variations du récit dans La Vie de Marianne de Marivaux, Paris, Klincksieck.

ROUSSET Jean (1962), Forme et signification. Essais sur les structures littéraires de Corneille à Claudel, Paris, José Corti.

ROUSSET Jean (1973), Narcisse romancier. Essai sur la première personne dans le roman, Paris, José Corti [notamment « Le miroir et l'autobiographie : La Vie de Marianne », p. 103-113].

ROUSSET Jean (1981), «L'échange divisé : La Vie de Marianne », in : Leurs yeux se rencontrèrent - la scène de première vue dans le roman, Paris, José Corti, p.115-121. 